\title{
Heavy water around the L1448-mm protostar
}

\author{
C. Codella ${ }^{1}$, C. Ceccarelli ${ }^{2}$, B. Nisini ${ }^{3}$, R. Bachiller ${ }^{4}$, J. Cernicharo ${ }^{5}$, F. Gueth ${ }^{6}$, A. Fuente ${ }^{4}$, and B. Lefloch ${ }^{2}$ \\ 1 INAF, Osservatorio Astrofisico di Arcetri, Largo E. Fermi 5, 50125 Firenze, Italy \\ e-mail: codella@arcetri.astro.it \\ 2 Laboratoire d'Astrophysique de l'Observatoire de Grenoble, BP 53, 38041 Grenoble Cedex, France \\ 3 INAF - Osservatorio Astronomico di Roma, via di Frascati 33, 00040 Monteporzio Catone, Italy \\ 4 Observatorio Astrónomico Nacional (IGN), Alfonso XII, 28014 Madrid, Spain \\ 5 Laboratory of Molecular Astrophysics, CAB (CSIC-INTA), Crta Ajalvir km 4, 28840 Madrid, Spain \\ 6 IRAM, 300 rue de la Piscine, 38406 Saint Martin d'Hères, France
}

Received 18 June 2010 / Accepted 13 October 2010

\section{ABSTRACT}

\begin{abstract}
Context. L1448-mm is the prototype of a low-mass Class 0 protostar driving a high-velocity jet. Given its bright $\mathrm{H}_{2} \mathrm{O}$ spectra observed with ISO, L1448-mm is an ideal laboratory to observe heavy water (HDO) emission.

Aims. Our aim is to image the HDO emission in the protostar surroundings, the possible occurrence of HDO emission also investigating off L1448-mm, towards the molecular outflow.

Methods. We carried out observations of L1448-mm in the $\operatorname{HDO}\left(1_{10}-1_{11}\right)$ line at $80.6 \mathrm{GHz}$, an excellent tracer of HDO column density, with the IRAM Plateau de Bure Interferometer.

Results. We image for the first time HDO emission around L1448-mm. The HDO structure reveals a main clump at velocities close to the ambient one towards the the continuum peak that is caused by the dust heated by the protostar. In addition, the HDO map shows tentative weaker emission at $\simeq 2000$ AU from the protostar towards the south, which is possibly associated with the walls of the outflow cavity opened by the protostellar wind.

Conclusions. Using an LVG code, modelling the density and temperature profile of the hot-corino, and adopting a gas temperature of $100 \mathrm{~K}$ and a density of $1.5 \times 10^{8} \mathrm{~cm}^{-3}$, we derive a beam diluted HDO column density of $\sim 7 \times 10^{13} \mathrm{~cm}^{-2}$, corresponding to a HDO abundance of $\sim 4 \times 10^{-7}$. In addition, the present map supports the scenario where HDO can be efficiently produced in shocked regions and not uniquely in hot corinos heated by the newly born star.
\end{abstract}

Key words. ISM: individual objects: L1448 - ISM: molecules - stars: formation

\section{Introduction}

Water can be considered as the most important molecule in the oxygen chemistry (after CO), influencing in particular the overall chemical composition of the gas involved in the process of star formation. In addition $\mathrm{H}_{2} \mathrm{O}$ is the most abundant component in the grain mantles and plays a fundamental role in the energy balance of star-forming regions (e.g., Ceccarelli et al. 1996; Kaufman \& Neufeld 1996; Bergin et al. 1998; van Dishoeck \& Blake 1998). In starless regions water abundance is very low $\left(\leq 10^{-8}\right.$, e.g., Bergin \& Snell 2002), while it increases to the abundance of CO $\left(\leq 10^{-4}\right)$ near protostars (e.g. Liseau et al. 1996; Harwit et al. 1998). Several mechanisms have been proposed for $\mathrm{H}_{2} \mathrm{O}$ formation (see the recent work on $\mathrm{H}_{2}{ }^{18} \mathrm{O}$ by Jørgensen \& van Dishoeck 2010, and reference therein): (i) heating from forming stars; (ii) jet-driven shocks; or (iii) shocks in accretion disks. In particular, water emission becomes even more important when studied in low-mass protostars, which are possibly associated with solar-type protoplanetary systems.

Unfortunately, the $\mathrm{H}_{2} \mathrm{O}$ low-energy lines are almost invisible from the ground due to very strong atmospheric absorption, and only under very special conditions are they observed towards the brightest sources (Cernicharo et al. 1990). As an example, $\mathrm{H}_{2} \mathrm{O}$ emission has been detected towards the low-mass protostar NGC 1333-IRAS 4B by different facilities, namely ISO, Odin, SWAS, Spitzer and, very recently, Herschel (Maret et al. 2002; Bjerkeli et al. 2009; Bergin et al. 2003; Franklin et al. 1989; Watson et al. 2007; Kristensen et al. 2010). These observations, with their relatively large apertures, revealed water from different components, namely the protostar, the outflow and the extended ambient medium. Recently Jørgensen \& van Dishoeck (2010) imaged IRAS 4B in the mm-range with the PdB interferometer. They detected $\mathrm{H}_{2} \mathrm{O}$ emission associated with the protostar, probably coming from the inner $25 \mathrm{AU}$ of the accretion disk, which calls for further observations of low-mass objects.

Given the difficulty of detecting water, several alternatives have been pursued. The most natural one is to observe rare isotopologues like HDO, which suffer much less telluric absorption and have some lines at sub-mm and mm-frequencies. Furthemore, if HDO is formed on the surfaces of grains before the heating due to the radiation from an accreting protostar (e.g., Parise et al. 2005a, and references therein), then its emission can also be used to trace the past history of water formation. Heavy water observations have been successfully carried out towards high-mass star-forming regions (Plambeck \& Wright 1987; Jacq et al. 1990; Gensheimer et al. 1996; Helmich et al. 1996; Pardo et al. 2001; Comito et al. 2003), but they are still very sparse. In Orion, the emission arises from warm gas $\left(T_{k}>150 \mathrm{~K}\right)$ and the abundance of HDO is strongly enhanced $\left(>10^{3}\right)$ with respect to ambient values, most likely because of recent HDO evaporation from dust grain mantles (see also Bergin et al. 2010, in the HIFI A\&A special issue). On the othe hand, the number of studies of HDO towards the lower-mass star-forming regions is even less. Stark et al. (2004) and Parise et al. (2005a) observed HDO emission with the JCMT and IRAM single-dish antennas, towards the hot corino heated by the IRAS 16293 protostar. In this case the measured deuteration ratio $\left(3 \times 10^{-2}\right)$ supports models where water is produced in the gas phase at low temperature and then storaged on the grains (e.g., Roberts et al. 2000). On 
the other hand, if $\mathrm{H}_{2} \mathrm{O}$ is formed in shocks, followed by condensation out on the grain mantles, the deuteration ratio is predicted to be lower than $10^{-3}$. It is still missing a study of HDO emission through interferometric high angular resolution images, which is needed to distinguish the hot-corino emission from that associated with more extended outflows.

In this Letter, we report for the first time a high-resolution image of HDO at $80.6 \mathrm{GHz}$ towards the protostar L1448-mm. We show that the emission at velocities close to the systemic one comes from the hot corino, we also report tentative HDO emission offset from the protostar, which is possibly associated with the cavity of the outflow red lobe.

\section{Observations and source selection}

L1448-mm, located at $250 \mathrm{pc}$ from the Sun, can be considered as the prototype of a low-luminosity $\left(\sim 8 L_{\odot}\right)$ Class 0 protostar. The newborn star is driving a highly collimated molecular jet consisting of well separated extremely high-velocity $\left(\geq 50 \mathrm{~km} \mathrm{~s}^{-1}\right)$ molecular bullets, well detected in $\mathrm{SiO}$ and $\mathrm{CO}$ (Guilloteau et al. 1992a; Bachiller et al. 1995). Lower velocity $\mathrm{CO}$ emission delineates a biconical cavity, possibly created by the passage of the jet, which is entraining the surrounding gas through successive bow-shock well detected in $\mathrm{H}_{2}$ (e.g., Davis \& Smith 1995; Dionatos et al. 2009; Neufeld et al. 2009). L1448-mm has been selected because of its bright $\mathrm{H}_{2} \mathrm{O}$ spectra that are observed with the ISO satellite, which lead to a large water abundance, $5 \times 10^{-4}$, towards the protostar (Nisini et al. 2000). The large ISO beam (80") does not allow one to exactly localise the $\mathrm{H}_{2} \mathrm{O}$-rich gas, but evidence is given that the high water abundance is maintained on a high level along the southern red-shifted part of the outflow.

The HDO observations of L1448-mm were obtained in July 2009 with the IRAM Plateau de Bure Interferometer (PdBI) in France. Four tracks in the five-element D configuration (baselines from $24 \mathrm{~m}$ up to $94 \mathrm{~m}$ ) were used, for a total time of $\sim 19 \mathrm{~h}$. A region of about $60^{\prime \prime}$ around the protostar has been mapped. The $\operatorname{HDO}\left(1_{10}-1_{11}\right)$ line at $80578.295 \mathrm{MHz}$ was observed with both 20 and $40 \mathrm{MHz}$ bandwidths and a corresponding spectral resolution of $\sim 0.13$ and $0.25 \mathrm{~km} \mathrm{~s}^{-1}$, respectively. Two other units with $320 \mathrm{MHz}$ bandwidth have been used to measure the continuum. We used the same setup for vertical and horizontal polarisation to gain in sensitivity. Amplitude and phase were calibrated by observing $0130-171,0234+285$ and $0333+321$, whereas the flux density scale was derived by observing $3 \mathrm{C} 84$ and $3 \mathrm{C} 454.3$, with an uncertainty of $\sim 20 \%$. Images were produced using natural weighting, and restored with a clean beam of $6 . .29 \times 4^{\prime \prime} .87\left(\mathrm{PA}=123^{\circ}\right)$. Note that we observed $\operatorname{HDO}\left(1_{10^{-}}\right.$ $\left.1_{11}\right)$ emission with a deep integration (230 min on-source) using the 30-m IRAM single-dish antenna and a HPBW of $31^{\prime \prime}$ (see Sect. 3).

\section{Results: the HDO maps}

Figure 1 shows the contour map of the $\operatorname{HDO}\left(1_{10}-1_{11}\right)$ integrated emission (contours) towards L1448-mm. The magenta contours trace the unresolved $3.7 \mathrm{~mm}$ continuum emission with a peak flux of $16 \mathrm{mJy}$ centred at $\alpha_{\mathrm{J} 2000}=03^{\mathrm{h}} 25^{\mathrm{m}} 38^{\mathrm{s}} .9, \delta_{\mathrm{J} 2000}=$ $+30^{\circ} 44^{\prime} 05^{\prime \prime}$. 1 , in agreement with previous observations (e.g. Guilloteau et al. 1992b; Bachiller et al. 1995). The HDO structure reveals a main clump (hereafter called A), (i) located close to the continuum peak due to the dust heated by the protostar and (ii) sligthly elongated in the S-E direction, i.e. the direction of the red-shifted outflow lobe. The offset seen in Fig. 1 between the the clump A and the continuum $\left(\leq 2^{\prime \prime}\right)$ is marginally significant, since the position uncertainty is around $1^{\prime \prime}$. Although we

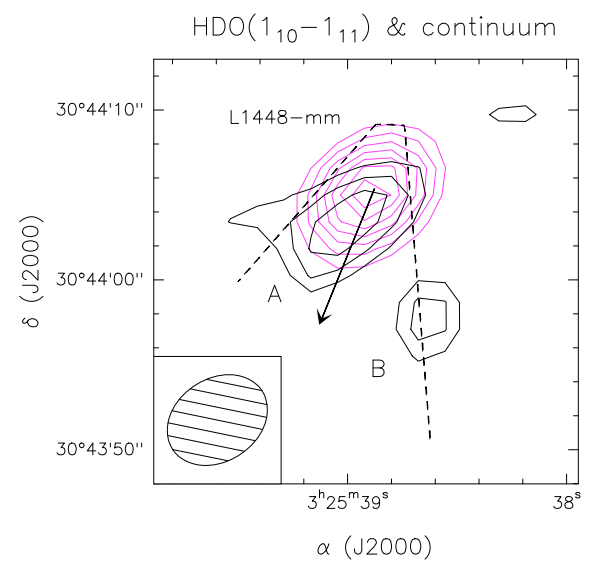

Fig. 1. Contour map of the $\operatorname{HDO}\left(1_{10}-1_{11}\right)$ emission (black contours) integrated between -0.3 and $+10.7 \mathrm{~km} \mathrm{ss}^{-1}$ superimposed onto the continuum image at $3.7 \mathrm{~mm}$ (magenta). The HDO contour levels range from $3 \sigma\left(33 \mathrm{mJy} \mathrm{km} \mathrm{s}^{-1}\right.$ beam $\left.^{-1}\right)$ to $5 \sigma$ by $1 \sigma$. Continuum contour levels range from $30(\sim 20 \sigma)$ to $90 \%$ of the peak value $(16 \mathrm{mJy})$, and identify the driving protostar L1448-mm. The filled ellipse in the lower left corner shows the synthesised PdBI beam (HPBW): 6.'29 $\times 4^{\prime \prime} .87$. The black arrow points in the direction of the jet $\left(\mathrm{PA}=-21^{\circ}\right)$ identified by Guilloteau et al. (1992) through SiO emission. The dashed lines identify the sketch of the limits of the conical blue-shifted $\left(+6,+8 \mathrm{~km} \mathrm{~s}^{-1}\right)$ cavity observed in $\mathrm{CO}(1-0)$ at PdBI by Bachiller et al. (1995).
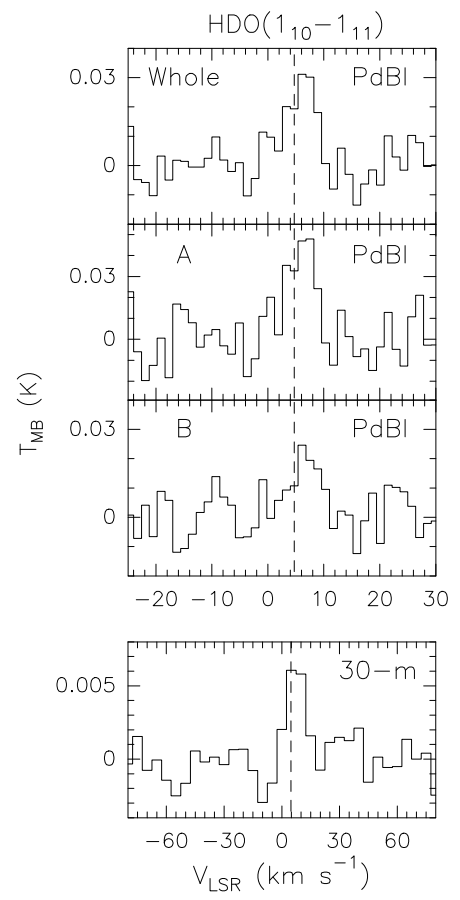

Fig. 2. $\operatorname{HDO}\left(1_{10}-1_{11}\right)$ spectra (in $T_{\mathrm{B}}$ scale) derived from the PdBI image and integrated over the whole emitting region (upper panel), and the area of the two structures called A and $\mathrm{B}$ (middle panels). The spectral resolution is $1.4 \mathrm{~km} \mathrm{~s}^{-1}$. Bottom panel reports the $\operatorname{HDO}\left(1_{10}-1_{11}\right)$ low-spectral resolution $\left(5 \mathrm{~km} \mathrm{~s}^{-1}\right)$ spectrum as observed with the $30-\mathrm{m}$ IRAM antenna. The ambient LSR velocity $\left(+4.7 \mathrm{~km} \mathrm{~s}^{-1}\right)$ is marked by the vertical dashed line.

cannot exclude a possible association of the clump A with the outflow (see below), we conservatively assume that clump A is tracing the hot-corino around the protostar. Beside clump A, the HDO map shows tentative emission from a weaker position (here called clump B) located at $\sim 8^{\prime \prime}(\simeq 2000 \mathrm{AU}$ at $250 \mathrm{pc})$ from the protostar, again towards the southern direction. No hints of HDO emission have been detected towards the $\mathrm{N}-\mathrm{W}$ direction, where the blue-shifted molecular lobe is located. 
Figure 2 shows the $\operatorname{HDO}\left(1_{10}-1_{11}\right)$ spectra, obtained from the PdBI maps by integrating over the whole area where heavy water emission is detected (upper panel) and over clumps A and B (middle panels). In particular, a Gaussian fit of the clump A spectrum leads to a peak of $49 \mathrm{mK}$ (in $T_{\mathrm{MB}}$ scale), observed with a $S / N=5$, at $+6.0 \pm 0.4 \mathrm{~km} \mathrm{~s}^{-1}$, i.e. slightly red-shifted with respect to the systemic velocity. The integrated intensity is $286 \pm$ $50 \mathrm{mK} \mathrm{km} \mathrm{s}^{-1}$, while the $F W H M$ linewidth is $5.7 \pm 1.0 \mathrm{~km} \mathrm{~s}^{-1}$. The spectra, smoothed to $1.4 \mathrm{~km} \mathrm{~s}^{-1}$ resolution to increase sensitivity, do not allow us a careful analysis of the line profile. Bottom panel of Fig. 2 reports the HDO spectrum as observed at low-spectral resolution $\left(5 \mathrm{~km} \mathrm{~s}^{-1}\right)$ after $230 \mathrm{~min}$ on-source using the 30-m IRAM antenna. The HDO line peaks at $\sim 6 \mathrm{mK}$ $(1 \sigma=0.2 \mathrm{mK})$. The line flux is $305 \mathrm{mJy} \mathrm{km} \mathrm{s}^{-1}$, showing that the PdB image collects about $61 \%\left(187 \mathrm{mJy} \mathrm{km} \mathrm{s}^{-1}\right.$ integrated over the 30-m beam) the of the flux observed with the IRAM single-dish. Given the calibration uncertainties of the PdBI image $(\sim 20 \%)$ and of the $30-\mathrm{m}$ measurement $(\leq 15 \%)$, the most plausible solution is that a weak extended structure has been filtered out by the interferometer.

We can compare the present HDO image with the $\mathrm{CO}(1-0)$ map obtained at the PdBI with a spatial resolution of $\sim 3^{\prime \prime}$ by Bachiller et al. (1995, see their Fig. 5). The dashed lines in Fig. 1 identify the position of the walls of the conical outflow cavity as observed at red-shifted velocities from +6 to $+10 \mathrm{~km} \mathrm{~s}^{-1}$. The black arrow shows the direction of the protostellar jet identified by Guilloteau et al. (1992) through $\mathrm{SiO}$ emission. Considering also the HDO spectrum of clump B, which shows emission at the same velocities, it is tempting to associate the HDO emission tentatively observed offset from the L1448-mm protostar for the first time with the cavity opened by the jet and well observed in $\mathrm{CO}$. Interestingly, one possible origin for the $\mathrm{H}_{2} \mathrm{O}$ masers at $22 \mathrm{GHz}$ observed at high-angular resolution studies towards low-mass protostars is its association with conical shells (Marvel et al. 2008), which may be caused by propagation of large bowshocks that entrain ambient medium at long transverse distances from the jet but which could also represent walls of evacuated cavities produced by a wide-angle wind (see e.g., Shang et al. 2007, and references therein). The water maser spots require extremely high-density $\left(\sim 10^{7}-10^{9} \mathrm{~cm}^{-3}\right.$, Elitzur et al. 1989; Kaufman \& Neufeld 1996) conditions, which could be found in compressed shocked layers at the interface with the surrounding medium. Indeed, Cernicharo et al. (1996) reported a spectrum at $183 \mathrm{GHz}$ observed with the 30-m IRAM antenna, which was owing to the $\mathrm{H}_{2} \mathrm{O}\left(3_{13}-2_{20}\right)$ transition. The $\mathrm{H}_{2} \mathrm{O}$ spectrum is composed by several narrow red-shifted components, resembling that classical maser spectral pattern. The brightest feature is at $\sim+9 \mathrm{~km} \mathrm{~s}^{-1}$, i.e. close the velocity observed with the present HDO emission, but the whole pattern covers a broad range of velocities, questioning a possible association of the $183 \mathrm{GHz}$ emission with the cavity walls.

In conclusion, the present HDO map supports the presence of water in the cavity walls and suggests that HDO can be found not only in heated hot cores/corinos, but also in shocked material.

\section{The HDO abundance inside and outside the hot corino}

To derive first the HDO column density in clump A and then the abundance, we used an LVG code with the collisional coefficients from Green (1989), in a plane parallel slab (where because the line is very thin optically the exact geometry is negligible). We considered a gas temperature in the 50-120 $\mathrm{K}$ range and a density between $1 \times 10^{7} \mathrm{~cm}^{-3}$ and $1 \times 10^{9} \mathrm{~cm}^{-3}$. Figure 3 shows the beam-averaged HDO column density corresponding to the

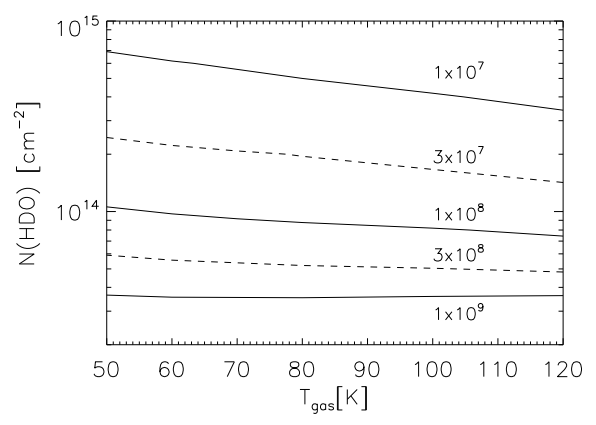

Fig. 3. Beam-averaged HDO total column density corresponding to the $\left(1_{10}-1_{11}\right)$ signal observed in the direction of the hot corino (clump A) as function of a gas temperature $T_{\text {gas }}$ between 50 and $120 \mathrm{~K}$ and for a density between $1 \times 10^{7} \mathrm{~cm}^{-3}$ and $1 \times 10^{9} \mathrm{~cm}^{-3}$. An LVG code in a plane parallel slab has been used (see Sect. 4).

signal observed in the direction of clump A as a function of the gas temperature for various densities. Within the $50-120 \mathrm{~K}$ interval of gas temperatures, the HDO total column density $\left(N_{\text {tot }}\right)$ varies very little as function of the gas temperature, and mostly depends on the gas density. At $100 \mathrm{~K}$, varying the density from $\sim 10^{8}$ to $\sim 10^{7} \mathrm{~cm}^{-3}$, corresponds to a factor $\sim 4$ in the HDO column density. At higher densities, the LTE solution is approached and the dependence on the gas density is not as strong.

If one assumes that the unresolved HDO emission towards the source is caused by the sublimation of the water ice mantles when the dust temperature reaches $100 \mathrm{~K}$, one can derive the abundance of the sublimated heavy water. We used the density and temperature profiles of the envelope of L1448-mm derived by Jørgensen et al. (2002) in order to evaluate the sizes and density of the sublimation region and its $\mathrm{H}_{2}$ column density. The sublimation region is predicted to have a radius of $\sim 25 \mathrm{AU}$, equivalent to a diameter of about $0^{\prime} .2$. At that radius, the $\mathrm{H}_{2}$ density is equal to $1.5 \times 10^{8} \mathrm{~cm}^{-3}$, giving an $\mathrm{H}_{2}$ column density of the sublimation region of about $1.1 \times 10^{23} \mathrm{~cm}^{-2}$. The beam-averaged HDO column density derived from the Fig. 3, assuming a density of $1.5 \times 10^{8} \mathrm{~cm}^{-3}$ and a temperature of $100 \mathrm{~K}$, is $\sim 7 \times 10^{13} \mathrm{~cm}^{-2}$. Thus, taking into account the dilution factor of the PdBI beam, the HDO abundance in the hot corino results in $\sim 4 \times 10^{-7}$. The main uncertainty in this value is due to the uncertainty in the radius, density and $\mathrm{H}_{2}$ column density of the sublimation region. A density a factor 3 larger would decrease the estimated HDO abundance by less than a factor 2, whereas a factor 3 lower density would increase the abundance by about a factor 4 . Taking the worst scenario, i.e. that the emission arises in a region of 5.'5 size, and using again the structure by Jørgensen et al. (2002), the emitting region would have a radius of $\sim 700 \mathrm{AU}$, where the gas temperature is $\sim 22 \mathrm{~K}$ and the density is $\sim 1.5 \times 10^{6} \mathrm{~cm}^{-3}$. The HDO column density $\left(\sim 1 \times 10^{16} \mathrm{~cm}^{-3}\right)$ and the abundance $\left(\sim 4 \times 10^{-7}\right)$ would be outrageously large for such a cold temperature, where water would be largely frozen onto the grain mantles, and we accordingly exclude this possibility.

The linewidth may bring some additional information. The observed HDO linewidth is around $6 \mathrm{~km} \mathrm{~s}^{-1}$. Again, following Jørgensen et al. (2002), if the emission originates in the assumed $25 \mathrm{AU}$, the mass of the central object is about $0.1 \mathrm{M}_{\odot}$. Alternatively, if the central object has a mass of $1 M_{\odot}$, the emission must originate in a region at about $100 \mathrm{AU}$, where the gas temperature is $\sim 50 \mathrm{~K}$, the density is $\sim 2 \times 10^{7} \mathrm{~cm}^{-3}$, and the $\mathrm{H}_{2}$ column density is $6 \times 10^{22} \mathrm{~cm}^{-2}$. The beam-averaged HDO column density would be $5 \times 10^{14} \mathrm{~cm}^{-2}$ (Fig. 3) and consequently the HDO abundance $\sim 5 \times 10^{-7}$.

In summary, the present observations suggest that the HDO abundance is $\sim 4 \times 10^{-7}$ and likely originates in a region whose 
radius is not much larger than about $100 \mathrm{AU}$. The most probable hypothesis is that the HDO emission observed in clump A is associated with the hot corino, and the sublimation of the icy mantles.

We also compared our observations with the theoretical predictions by Parise et al. (2005b), who carried out a theoretical study of the HDO emission in L1448-mm. Their Fig. B2, upper right panel, shows the dependence of the HDO flux as function of the HDO abundance in the outer envelope (where the dust temperature is less than $100 \mathrm{~K}$ ) and in the hot corino. The curves are degenerate, in the sense that the observed signal is obtained either when (1) the HDO abundance is $\sim 2 \times 10^{-8}$ in the outer envelope and lower than $\sim 5 \times 10^{-8}$ in the hot corino or (2) the HDO abundance is $\sim 5 \times 10^{-7}$ in the hot corino and lower than $\sim 3 \times 10^{-9}$ in the outer envelope. The present interferometric observations suggests that the emission observed towards L1448-mm originates in the hot corino, thus solving the degeneracy. Also, note that the abundance derived in our simple analysis agrees very well with the abundance derived using this more sophisticate modelling.

As discussed in the introduction, HDO can be a useful probe of the corresponding main isotopologue, $\mathrm{H}_{2} \mathrm{O}$, which is largely undetectable from ground. The study of the water deuteration in a similar solar type protostar, IRAS 16293-2422, by Parise et al. (2005a) suggested that the $\mathrm{HDO} / \mathrm{H}_{2} \mathrm{O}$ ratio in the sublimated ices is around 3\%. When comparing L1448-mm and IRAS 16293-2422 with other sources where the molecular deuteration of formaldehyde and methanol was measured, it appears that both L1448-mm and IRAS 16293-2422 are typical sources (Parise et al. 2006). It is then plausible that the $\mathrm{HDO} / \mathrm{H}_{2} \mathrm{O}$ in L1448-mm is similar to what was found in IRAS 16293-2422, namely $\sim 3 \%$, implying a $\mathrm{H}_{2} \mathrm{O}$ abundance of about $\sim 1 \times 10^{-5}$. This is somewhat similar to the water abundance estimated in IRAS 16293-2422 by ISO observations (Ceccarelli et al. 2000; Crimier et al. 2006), and troubling because it would imply either an abundance of the water ices lower than usually assumed, $\sim 10^{-4}$, or that not all ices have been sublimated. New observations by the just launched Herschel satellite will soon confirm or disregard this result and clarify the situation.

The HDO emission tentatively observed outside the hot corino and presumibly associated with the outflow red-shifted cavity observed in $\mathrm{CO}$ is only a factor 2 weaker than that observed towards the protostar (see HDO spectra of clumps A and $\mathrm{B}$ reported in Fig. 2). Therefore, as for the hot corino region, this HDO signal corresponds to a HDO column density of about $10^{14} \mathrm{~cm}^{-2}$ if the density is around $10^{8} \mathrm{~cm}^{-3}$ and the emission fills up the PdB beam. It is difficult to estimate the abundance of HDO because of the lack of available estimates of the $\mathrm{H}_{2}$ emission in the cavity. Even though we are unable to quantify how much water has been sputtered or sublimated from the grain mantles, clearly one or more shocks are responsible for the HDO spot that is coincident with the cavity wall. In other words, HDO emission cannot be just limb-brightned emission: water must have been extracted from the grain mantles by some energetic process. As previously noted by other authors in other contexts, models of bow shock naturally would explain the morphology and the presence of shock "far away" from the high-velocity jet.

\section{Conclusions}

The present high spatial resolution map of L1448-mm allows us to image for the first time HDO emission at $80.6 \mathrm{GHz}$ around a Class 0 protostar with a spatial scale of 5'.5, i.e. $\sim 1400 \mathrm{AU}$ at $250 \mathrm{pc}$. We have shown that the bulk of the emission at velocities close to the ambient one likely comes from the hot corino, whereas a tentative detection of HDO emission is present at $\sim 2000$ AU from the protostar, and it is possibly associated with the walls of the outflow cavity previously observed in CO. These findings support the idea that HDO can be efficiently produced in shocked regions and not uniquely in hot cores heated by the newly born star.

We derived the HDO abundance in the hot corino with the reconstruction of the density and temperature profiles by Jørgensen et al. (2002). Adopting a gas temperature of $100 \mathrm{~K}$ and a density of $1.5 \times 10^{8} \mathrm{~cm}^{-3}$, a HDO column density of $\sim 7 \times 10^{13} \mathrm{~cm}^{-2}$ was derived, corresponding to a HDO abundance of $\sim 4 \times 10^{-7}$. If we assume that the $\mathrm{HDO} / \mathrm{H}_{2} \mathrm{O}$ in $\mathrm{L} 1448-\mathrm{mm}$ is similar to what was found in IRAS 16293-2422 ( 3\%), a $\mathrm{H}_{2} \mathrm{O}$ abundance of about $10^{-5}$ is derived. Only future estimates of the $\mathrm{H}_{2}$ column density will allow us to give an estimate of the HDO abundance in the outflow.

Acknowledgements. We thank S. Cabrit, M. Tafalla, and J. Santiago-García for fruitful discussions and suggestions. We also thank an anonymous referee for detailed comments that have greatly helped to improve this paper.

\section{References}

Bachiller, R., Guilloteau, S., Dutrey, A. P., Planesas, P., \& Martín-Pintado, J. 1995, A\&A, 299, 857

Bjerkeli, P., Liseau, R., Olberg, M., et al. 2009, A\&A, 507, 1455 Bergin, E. A., \& Snell, R. L. 2002, ApJ, 581, L105

Bergin, E. A., Neufeld, D., \& Melnick, G. J. 1998, ApJ, 499, 777

Bergin, E. A., Neufeld, D. A., \& Melnick, G. J. 1999, ApJ, 510, L45

Bergin, E. A., Kaufman, M. J., Melnick, G. J., Snell, R. L., \& Howe, J. E. 2003, ApJ, 582, 830

Bergin, E. A., Hogerheijde, M. R., Brinch, C., et al. 2010, A\&A, 521, L33

Ceccarelli, C., Hollenbach, D. J., \& Tielens, A. G. G. M. 1996, ApJ, 471, 400

Ceccarelli, C., Castets, A., Caux, E., et al. 2000, A\&A, 355, 1129

Ceccarelli, C., Maret, S., Tielens, A. G. G. M., Castets, A., \& Caux, E. 2003, A\&A, 410, 587

Cernicharo, J., Thum, C., Hein, H., et al. 1990, A\&A, 231, L15

Cernicharo, J., Bachiller, R., \& González-Alfonso, E. 1996, A\&A, 305, L5

Comito, C., Schilke, P., Gerin, M., et al. 2003, A\&A, 402, 635

Crimier, N., Ceccarelli, C., Lefloch, B., \& Faure, A. 2009, A\&A, 506, 1229

Davis, C. J., \& Smith, M. D. 1995, ApJ, 443, L41

Dionatos, O., Nisini, B., \& Garcia Lopez, R. 2009, ApJ, 692, 1D

Elitzur, M., Hollenbach, D. J., \& Mackee, C. F. 1989, ApJ, 346, 983

Franklin, J., Snell, R. L., Kaufman, M. J., et al. 2008, ApJ, 674, 1015

Gensheimer, P. D., Mauersberger, R., \& Wilson, T. L. 1996, A\&A, 314, 281

Giannini, T., Nisini, B., \& Lorenzetti, D. 2001, ApJ, 555, 40

Green, S. 1989, ApJS, 70, 813

Guilloteau, S., Delannoy, J., Downes, D., et al. 1992a, A\&A, 262, 624

Guilloteau, S., Bachiller, R., Fuente, A., \& Lucas, R. 1992b, A\&A, 265, L49

Harwit, M., Neufeld, D. A., Melnick, G. J., \& Kaufman, M. J. 1998, ApJ, 497, L105

Helmich, F., van Dishoeck, E. F., Blake, G. A., et al. 1996, A\&A, 315, L173

Jacq, T., Walmsley, C. M., Henkel, C., et al. 1990, A\&A 228, 447

Jørgensen, J. K., \& van Dishoeck, E. F. 2010, ApJ, 710, L72

Jørgensen, J. K., Schöier, F. L., \& van Dishoeck, E. F. 2002, A\&A, 389, 908

Kaufman, K. J., \& Neufeld, D. 1996, ApJ 456, 611

Kristensen, L. E., Visser, R., van Dishoeck, E. F., et al. 2010, A\&A, 521, L30

Liseau, R., Ceccarelli, C., Larsson, B., et al. 1996, A\&A, 315, L181

Maret, S., Ceccarelli, C., Caux, E., Tielens, A. G. G. M., \& Castets, A. 2002, A\&A, 395, 573

Marvel, K. B., Wilking, B. A., Claussen, M. J., \& Wootten, A. 2008, ApJ, 685, 285

Neufeld, D. A., Nisini, B., Giannini, T., et al. 2009, ApJ, 706, 170

Nisini, B., Benedettini, M., Giannini, T., et al. 2000, A\&A, 360, 297

Pardo, J. R., Cernicharo, J., Herpin, F., et al. 2001, ApJ, 562, 799

Parise, B., Caux, E., Castets, A., et al. 2005a, A\&A, 431, 547

Parise, B., Ceccarelli, C., \& Maret, S. 2005b, A\&A, 441, 171

Parise, B., Ceccarelli, C., Tielens, A. G. G. M., et al. 2006, A\&A, 453, 949

Plambeck, R. L., \& Wright, M. 1987, ApJ, 317, L101

Roberts, H., \& Millar, T. 2000, A\&A, 374, 780

Shang, H., Li, Z.-Y., \& Hirano, N. 2007, in Protostars and Planets V, ed. B.

Reipurth, D. Jewitt, \& K. Keil, 261

Stark, R., Sandell, G., Beck, S., et al. 2004, ApJ, 608, 341

van Dishoeck, E. F., \& Blake, G. A. 1998, ARA\&A, 36, 317

Watson, D. M., Bohac, C. J., Hull, C., et al. 2007, Nature, 448, 1026 